\title{
Search for a new type of spatial organization of nucleic acids in human genome
}

\author{
Zamoskovtseva A.A. ${ }^{1,2 *}$, Lomzov A.A. ${ }^{1,2}$, Kabilov M.R. ${ }^{1}$, Pyshnyi D.V. ${ }^{1}$ \\ ${ }^{1}$ Institute of Chemical Biology and Fundamental Medicine SB RAS, Novosibirsk, Russia \\ ${ }^{2}$ Novosibirsk State University, Novosibirsk, Russia \\ *e-mail: zamosk_n@mail.ru
}

Key words: nucleic acids, pseudoknots, bioinformatic algorithm, human genome

Motivation and Aim: One of the functions of nucleic acids is regulation of various biological processes. In many ways, it is determined by the secondary and tertiary structures. Recently, a new type of spatial organization of nucleic acids was discovered in the ICBFM SB RAS. This self-limited complex contains two duplex fragments and single-stranded loops between them. It is called a TW-type pseudoknot (twiplex). It can be formed by single or two DNA (or RNA) chain, or in DNA/RNA intermolecular complex. The aim of the work is to determine the possibility of implementing TW-type pseudoknots with DNA chains of living systems.

Methods and Algorithms: To do this we searched for such sequences in the human genome using the home made Python scripts and proceed analysis using by RNAstructure program [1] to select sequences for further analysis. To verify experimentally the formation of TW-type pseudoknots by selected sequences we used a number of experimental methods such as thermal denaturation with optical signal registration, gel shift assay and the atomic force microscopy. Biological role analysis of such structures in the human genome was analyzed with the help of BLAST [2] and BEDTools [3].

Results: We suggested conditions required for twiplex formation such as oligonucleotide length limitation. Given the formulated conditions, an algorithm was developed for bioinformatic search on sequences which potentially can form twiplex, and on its basis a script in Python was written and applied for the human genome analysis. Structures with double-stranded blocks of the length from 5 to 20 base pairs are considered. Genome regions potentially capable for the twiplexes formation have been detected. The most represented structure which occurs more than 200 times in our sample and which is evenly distributed across all chromosomes, was chosen for further experimental analysis. The formation a TW-type pseudoknots by single DNA strand was confirmed using a model system by a gel-shift assay method. The location of the founded sequences in genes, introns or exons was analyzed. Experimental studies have begun to define the physicochemical and molecular biological properties of selected nucleotide sequences.

Acknowledgements: Supported by the RFBR (20-04-00719) and by State project no. A-0309-2016-0004.

\section{References}

1. Reuter JS, Mathews DH. RNAstructure: software for RNA secondary structure prediction and analysis. $B M C$ Bioinformatics. 2010;11:129. Published 2010 Mar 15.

2. Altschul SF, Gish W, Miller W, Myers EW, Lipman DJ. Basic local alignment search tool. J Mol Biol. 1990;215(3):403410. doi:10.1016/S0022-2836(05)80360-2.

3. Aaron R. Quinlan, Ira M. Hall, BEDTools: a flexible suite of utilities for comparing genomic features, Bioinformatics, V. 26, Issue 6, 15 March 2010, P.841-842. 